\title{
Deficiência mental: o que dizem os manuais diagnósticos?
}

\author{
Fabiane Romano de Souza Bridi* \\ Claudio Roberto Baptista**
}

\section{Resumo}

Este trabalho surge da necessidade de se investigar o que dizem os manuais diagnósticos acerca da deficiência mental, principalmente, no que se refere ao conceito de deficiência mental, aos critérios de diagnóstico e classificação. Na presente pesquisa trabalhou-se com: o Manual Diagnóstico e Estatístico de Transtornos Mentais - DSM-IV (2002); a Classificação Internacional das Doenças - CID-10, (OMS, 1993); a Classificação Internacional de Funcionalidade, Incapacidade e Saúde - CIF, (OMS, 2003); e com Retardo mental: definição, classificação e sistemas de apoio (AAMR, 2002). Os pressupostos teóricos do pensamento sistêmico serviram de ferramenta analítica do material investigado. Foi possível observar uma estreita ligação entre a proposição teórica dos diferentes manuais. Apesar da existência de algumas diferenças, essas mudanças não chegam a produzir uma ruptura epistemológica nas formas de se conceber e identificar o fenômeno em questão, apresentando-se filiada aos pressupostos tradicionais do pensamento científico. Entende-se que a compreensão teórico-conceitual a respeito do fenômeno da deficiência mental traz implicações diretas na proposição e nas configurações das práticas pedagógicas, justificando-se, desta forma, realização desse estudo.

Palavras-chave: Educação Especial; Deficiência mental; Manuais diagnósticos.

\footnotetext{
* Professora Doutora da Universidade Federal de Santa Maria, Santa Maria, Rio Grande do Sul, Brasil.

** Professor Doutor da Universidade Federal do Rio Grande do Sul, Porto Alegre, Rio Grande do Sul, Brasil.
} 


\section{Mental retardation: What do the diagnostic manuals say about it?}

\section{Abstract}

This work emerges from the need to investigate what the diagnostic manuals say about mental retardation, especially with regard to the concept of mental retardation, the criteria for diagnosis and its classification. In the present study, we worked with: Manual Diagnóstico e Estatístico de Transtornos Mentais - DSM-IV (2002); Classificação Internacional das Doenças - CID-10, (OMS, 1993); Classificação Internacional de Funcionalidade, Incapacidade e Saúde - CIF, (OMS, 2003) and Retardo mental: definição, classificação e sistemas de apoio (AAMR, 2002). The theoretical assumptions of systemic thinking served as an analitical tool of the material investigated. It was possible to observe a close connection among the theoretical proposition of the different manuals. Although, there are some differences, theses changes do not amount to produce an epistemological rupture in the ways of conceiving and identifying the phenomenon in question, presenting affiliated to traditional assumptions of scientific thinking. It is undestood that the theoretical and conceptual understanding about the phenomenon of mental retardation have direct implications on the proposition and configurations of pedagogical practices, justifying, thus, this study.

Keywords: Special Education; Mental retardation; Diagnostic manuals.

\section{Diálogos iniciais}

Abordar os manuais diagnósticos acerca da deficiência mental remete inevitavelmente às relações históricas entre a medicina e a educação especial, e, principalmente, aos modos como a primeira influencia, determina e delimita a segunda.

$\mathrm{O}$ ato de diagnosticar surge a partir da medicina. Inicialmente, é este campo do saber responsável pelo conceito e pela prática diagnóstica. Moysés (2008), a partir dos estudos foucaultianos, percorre os caminhos da medicina que a instituem como ciência, demonstrando como seus domínios científicos invadem e estendem-se para outras áreas de conhecimento.

A medicina constitui as raízes epistemológicas de todas as ciências da saúde, que dela se derivam e são filiadas, aparentadas. Sua importância, nesse sentido, extrapola, porém, o campo da saúde; ao construir o primeiro discurso científico sobre o indivíduo, ao ser ciência que toma o homem como objeto de saber positivo, torna-se raiz constituinte de todas as ciências do homem. (MOYSÉS, 2008, p. 142)

Dessa forma, temos uma presença e uma sobreposição do saber médico sobre os outros campos disciplinares, incluindo a educação, pautada a partir dos conceitos de normalidade e desviante (MOYSÉS, 2008), ou normal e patológico (VASQUES, 
2008), a medicina, por meio do seu olhar clínico, realiza a ação de classificar, de decidir, de enquadrar. "O olhar clínico simboliza um olhar que sabe e decide, portanto pode reger. Para se constituir como tal, precisa aprender a ver, isolar, reconhecer diferenças e semelhanças, agrupar, classificar." (MOYSÉS, 2008, p. 158). É esse olhar que produz o diagnóstico; sua extensão perpassa a medicina e constitui outras disciplinas que se ocupam do humano, como a psicologia.

Originário da palavra grega diagnóstikos, significa conhecer, discernir. O diagnóstico seria o conhecimento ou determinação de uma doença pela observação de seus sintomas. Os fatos que iluminam uma conclusão. O primeiro passo do processo terapêutico. O momento em que uma decisão sobre normalidade e patologia deve ser tomada. Uma pergunta que balança os alicerces do conhecimento. Uma encruzilhada. Em que direção apontar o tratamento e a escolarização? (VASQUES, 2009, p. 108)

Os processos diagnósticos, no campo da deficiência mental, cabe destacar, historicamente, têm sido realizados pela medicina e pela psicologia. Quando produzidos a partir da medicina, referem concepções organicistas de deficiência, com ênfase na dimensão biológica associada à etiologia. Quando produzidos a partir da psicologia, caracterizam-se por serem diagnósticos psicométricos. Ambos os caminhos diagnósticos geram prognósticos muito fechados; são mapas restritos e previsíveis sobre os sujeitos, com pouco espaço para a surpresa e para o novo e, nessa medida, produzem o estático, a impossibilidade do vir a ser.

Cabe situar que, ao longo deste trabalho, ao mencionar o diagnóstico clínico da deficiência mental, estarei me referindo ao diagnóstico que pode ser produzido pela medicina e, assim, gerado a partir de uma perspectiva organicista, ou produzido no campo da psicologia, a partir de uma perspectiva psicométrica. Ambas as propostas são associadas a uma perspectiva de compreensão da deficiência que coloca o foco no sujeito como referência fundamental para a compreensão de suas 'faltas'. Esse é o eixo organizador das duas proposições: a ausência de contextualização, e o precário lugar destinado às relações como produtoras do sujeito.

Estudos atuais têm apontado a perspectiva clínica como sustentação tanto das propostas de formação de professores (MICHELS 2009a, 2009b), como na organização do trabalho pedagógico. É observado um privilégio num “...modus operandi clínico, com foco especializado, em desequilíbrio com os processos pedagógicos e que se desenvolve de forma paralela, com certa autonomia, em relação à educação formal". (GARCIA, 2009a, p. 1).

\section{A deficiência mental nos manuais diagnósticos}

Os diagnósticos clínicos de deficiência mental têm sido realizados com base nos manuais internacionais de classificação das doenças, como o Manual Diagnóstico e Estatístico de Transtornos Mentais (DSM-IV), publicado pela Associação Psiquiátrica Americana; a Classificação Internacional das Doenças (CID-10), publicada pela Organização Mundial da Saúde (OMS), e a Classificação Internacional de Funcionalidade, Incapacidade e Saúde (CIF), também publicada pela Organização Mundial de 
Saúde (OMS) em 2001. Os sistemas CID-10 e CIF são reconhecidos pela OMS como sistemas complementares, na medida em que a informação sobre o diagnóstico (CID10) somada à funcionalidade (CIF) proporciona um quadro mais amplo sobre a saúde e as possibilidades do indivíduo. No campo mais específico da deficiência mental, temos o sistema de classificação proposto pela Associação Americana de Retardo Mental (AAMR) por meio da publicação Retardo mental: definição, classificação e sistemas de apoio, também conhecido como Sistema $2002^{1}$.

A deficiência mental, nos manuais - DSM-IV, CID-10 e Sistema 2002 -, aparece sob a nomeação de retardo mental. Nos três manuais, o retardo mental implica um funcionamento intelectual significativamente abaixo da média e comprometimento no comportamento adaptativo, tendo seu início antes dos 18 anos de idade. A presença desses três critérios é necessária para se pensar na hipótese diagnóstica.

Retardo mental é uma incapacidade caracterizada por importantes limitações, tanto no funcionamento intelectual quanto no comportamento adaptativo; está expresso nas habilidades adaptativas conceituais, sociais e práticas. Essa incapacidade tem início antes dos 18 anos." (AAMR, 2006, p. 20)

Merecem destaques os instrumentos a serem utilizados para a constatação das evidências indicadoras de um diagnóstico de deficiência mental. Para verificar um funcionamento intelectual significativamente abaixo da média, está prevista, nos três manuais, a utilização dos testes de quociente de inteligência (QI)².

O surgimento das medidas de inteligência teve relação direta com o campo escolar, a partir das contribuições psicométricas, principalmente, de Alfred Binet, no início do século XX. A psicometria surge e desenvolve-se no cerne da psicologia escolar. Esta última tem como precursor Galton, que, junto com Cattell e Binet, formam as "bases do tripé sobre o qual se constrói a psicologia escolar, fundada nos estudos das diferenças individuais." (MOYSÉS, 2008, p. 100)

Francis Galton (1822-1911)3, influenciado pela teoria evolucionista de Darwin, "foi o primeiro a fazer o transplante dos princípios evolucionistas de variação, seleção e adaptação para o estudo das capacidades humanas." (PATTO, 2010, p. 60). Objetivou a seleção dos psicologicamente mais capazes com o propósito de aprimoramento da espécie humana. Segundo Moysés (2008), em seu Laboratório de Psicometria, no University College, de Londres, desenvolveu instrumentos com o propósito de mensurar as diferenças individuais.

A difusão das ideias de Galton influenciou fortemente a proposição dos testes de Inteligência; entre seus alunos esteve Cattell, considerado um dos pioneiros na criação dos testes psicológicos e fundador do movimento norte-americano dos testes. Galton foi o primeiro a usar "mental tests" para referir instrumentos que permitissem a mensuração de uma função mental específica.

Porém, o grande impacto e a significativa difusão dos testes de inteligência ocorrem por meio das contribuições de Alfred Binet. Este psicólogo publica o primeiro teste de inteligência, conhecido com o teste Binet-Simon, elaborado por ele e o 
médico Theodore Simon. A referida testagem foi estruturada a partir da observação de suas duas filhas e compreendia a realização de tarefas intelectuais para possibilitar a identificação da idade mental das crianças.

A proposição inicial deste psicólogo surge da sua preocupação em relação aos alunos que não conseguiam aprender nas escolas públicas de Paris. Parte do pressuposto de que a inteligência não havia se desenvolvido de forma satisfatória. Binet propõe a construção de um instrumento diagnóstico capaz de medir a inteligência das crianças, e, a partir deste, decidir qual a intervenção educacional mais apropriada a cada uma delas. Dessa forma, com o objetivo de conhecer o potencial global da criança, construiu uma ampla série de tarefas organizadas por grau de dificuldade, em busca de um valor numérico capaz de representá-lo.

Os resultados da idade mental que Binet encontrava tendiam a se correlacionar com as opiniões dos professores sobre a inteligência de seus alunos. Uma coincidência nada surpreendente, uma vez que a maioria das tarefas propostas em seu teste apresentavam grande semelhança com o que era enfatizado no sistema escolar francês. Se se lembrar a preocupação de Binet, pode-se considerar adequada essa similaridade de tarefas, pois pretendia instrumentalizar a identificação de crianças que não iriam se beneficiar da educação escolar, com o intuito de submetê-las a procedimentos de ortopedia mental, que aumentassem sua inteligência. Se se pretende identificar crianças que irão fracassar no sistema formal de educação, é coerente usar tarefas de mesma ordem que as propostas pela escola... (MOYSÉS, 2008, p. 101)

A proposta de medição do quociente de inteligência de Binet, posteriormente, constitui-se em escala de medida de inteligência (atualmente conhecida como Stanford-Binet), permitindo o estabelecimento de comparações entre o desenvolvimento "normal" e o "atrasado". Com esse trabalho, Binet oferece elementos para o diagnóstico psicológico da deficiência mental, ao mesmo tempo em que a deficiência mental deixa de ser uma atribuição somente da medicina e passa a pertencer à psicologia como questão teórica. Os efeitos dessa proposta de medição são observados em classificações e rotulações de deficiente mental e/ou limítrofe, usados para designar a incapacidade intelectual dos sujeitos que não correspondem às medidas padrão dos testes de inteligência. (PESSOTTI, 1984; CARNEIRO, 2006 e 2008)

Atualmente, as escalas de medição da inteligência ainda são amplamente usadas - inclusive por serem propostas como instrumentos diagnósticos nos manuais classificatórios - apesar das críticas e problematizações quanto a sua utilização. "É interessante observar como o QI permanece central à definição, quase um século após ser "descoberto", a despeito do acúmulo de críticas a sua utilização para definir e diagnosticar a deficiência." (NUNES; FERREIRA, 1993, p. 38). As críticas centram-se tanto no uso dos testes de inteligência na definição e diagnóstico da deficiência mental quanto nas possibilidades de elaboração de prognósticos sobre a vida do sujeito e sua escolarização. 
As classificações por nível de QI expressam uma visão psicométrica da deficiência e apontam prognósticos desanimadores. O foco do problema é colocado no sujeito, no seu desempenho, consolidando o atraso cognitivo como característica individual, contribuindo para uma baixa expectativa dos professores em relação a esse aluno. (CARNEIRO, 2008, p. 20)

O comprometimento no comportamento adaptativo é verificado com base na utilização de escalas de maturação e de comportamento ${ }^{4}$. Tais escalas objetivam o conhecimento e a verificação do comportamento adaptativo em três dimensões: conceituais, sociais e práticas. Objetivam, ainda, conhecer as relações entre estas três dimensões e o seu funcionamento no cotidiano dos sujeitos. As habilidades conceituais compreendem, por exemplo, o conhecimento e a utilização da linguagem, da leitura e da escrita, dos conceitos de dinheiro, do autodirecionamento (AARM, 2006). As habilidades sociais envolvem o relacionamento interpessoal, a responsabilidade, a autoestima, a credibilidade (ou seja, a probabilidade de ser enganado ou manipulado), a ingenuidade, a capacidade de se submeter às regras, de obedecer às leis e evitar a vitimização (AARM, 2006). E as habilidades práticas compreendem as atividades de vida diária (como a mobilidade, o comer, usar o banheiro, vestir-se), atividades instrumentais da vida diária (preparar refeições, cuidar da casa, transportar-se, tomar remédio, lidar com o dinheiro, usar telefone), as habilidades ocupacionais e a manutenção dos ambientes seguros (AAMR, 2006). Apesar de a utilização dessas escalas estarem previstas nos três manuais, visando ao conhecimento do comportamento adaptativo, cabe destacar que, segundo Almeida (2004), nenhuma delas tem validação para o território nacional, trazendo efeitos, na opinião da autora, aos processos diagnósticos no campo da deficiência mental.

A referida autora sinaliza a estreita relação entre os três diferentes manuais de classificação - CID-10, DSM-IV e Sistema 2002 - afirmando que essa sintônica relação está presente desde 1983, quando os autores AAMR participaram na definição do conceito de deficiência mental nos outros manuais classificatórios CID-9 e DSM-III, com o objetivo de tornarem tais sistemas mais compatíveis.

Carvalho e Maciel (2003) também afirmam a importante participação da Associação Americana de Retardo Mental (AARM), fundada em 1876, na construção do conceito de deficiência mental e sua influência nos outros manuais classificatórios. As referidas autoras ainda observam o fato de o Sistema 2002 não ser tão conhecido e difundido no Brasil em comparação aos outros sistemas classificatórios; talvez, por sua pouca inserção nas produções e na literatura específica no campo da deficiência mental. Além disso, a décima edição do manual - Retardo mental: definição, classificação e sistemas de apoio - tem sua primeira publicação, em nosso país, em 2006, fato que também corrobora o seu restrito conhecimento.

Quanto às relações entre as atuais edições dos diferentes manuais classificatórios, o próprio Sistema 2002 discute sua relação com outros sistemas de classificação (DSM-IV-TR, CID-10 e CIF), estabelecendo suas semelhanças e diferenças (ALMEIDA, 2004). 
No que se refere à Classificação Internacional de Funcionalidade, Incapacidade e Saúde (CIF), como instrumento de classificação, ela é complementar a CID-10, usada na realização dos diagnósticos. A CIF é utilizada para conhecer a funcionalidade do sujeito, vai além de uma perspectiva médica e inclui as dimensões individuais e sociais. A CIF, apesar de não se referir especificamente à deficiência mental, traz efeitos neste campo por redimensionar o conceito de deficiência. Segundo Diniz, Medeiros e Squinca (2007), foram 20 anos de debates entre o modelo biomédico e o social de deficiência, resultando na revisão do modelo classificatório proposto pela OMS culminado na CIF. Dessa forma, a CIF referencia os dois modelos de deficiência - biomédico e social - e propõe a adoção do modelo biopsicossocial, resultado da combinação de ambos.

A CIF é baseada, portanto, numa abordagem biopsicossocial que incorpora os componentes de saúde nos níveis corporais e sociais. Assim, na avaliação de uma pessoa com deficiência, esse modelo destaca-se do biomédico, baseado no diagnóstico etiológico da disfunção, evoluindo para um modelo que incorpora as três dimensões: a biomédica, a psicológica (dimensão individual) e a social. Nesse modelo cada nível age sobre e sofre a ação dos demais, sendo todos influenciados pelos fatores ambientais. (FARIAS; BUCHALLA, 2005, p. 189)

O modelo da CIF se propõe à substituição de uma perspectiva reducionista da deficiência e da incapacidade por um enfoque mais amplo e possibilitador, o qual considera, apesar das alterações de função e de estrutura apresentadas, a participação social do indivíduo e as atividades por este desempenhadas. Este modelo compreende a funcionalidade e a incapacidade como dimensões determinadas pelo contexto e pelo ambiente.

Segundo a AAMR (2006), o modelo da CIF encontra pontos de aproximação com a proposição da AAMR, na medida em que os dois modelos propõem o conceito de deficiência a partir do funcionamento humano e das interações da pessoa com o ambiente. Aproximam-se, os dois modelos, ao assumirem o caráter dinâmico em que a deficiência não implica um estado permanente, podendo apresentar alterações e mudanças ao longo do tempo; ao basearem sua avaliação no funcionamento, reconhecem pontos incentivadores e inibidores deste funcionamento, e, por fim, enfocam uma orientação a qual privilegia as possibilidades ao observar como os recursos do ambiente podem ajudar a melhorar a vida das pessoas com deficiência.

Quanto à proposição do Sistema 2002, para a aplicação da definição de deficiência mental, devem ser consideradas cinco hipóteses:

1. as limitações no funcionamento atual devem ser consideradas dentro do contexto dos ambientes da comunidade característicos das pessoas da mesma faixa etária e da mesma cultura do indivíduo;

2. a avaliação válida considera a diversidade cultural e linguística e também as diferenças na comunicação, nos fatores sensoriais, motores e comportamentais; 
3. em cada indivíduo, as limitações frequentemente coexistem com as potencialidades;

4. um propósito importante, ao descrever as limitações, é o de desenvolver um perfil de apoios necessários;

5. com apoios personalizados apropriados, durante um determinado período de tempo, o funcionamento cotidiano da pessoa com retardo mental em geral melhora. (AAMR, 2006, p. 25)

Esta definição é caracterizada por uma concepção multidimensional da deficiência mental, propondo cinco dimensões para a compreensão do conceito: Dimensão I - Habilidades Intelectuais; Dimensão II - Comportamento Adaptativo; Dimensão III - Participação, Interações e Papéis Sociais; Dimensão IV - Saúde e Dimensão V Contextos. (AARM, 2006).

A Dimensão I - Habilidades Intelectuais - compreende a inteligência como uma competência geral. Envolve o raciocínio, o planejamento, a resolução de problemas, o pensamento abstrato, a compreensão de ideias complexas, a rapidez no processo de aprendizagem e a aprendizagem pela experiência. As habilidades intelectuais são avaliadas objetivamente por meio dos testes de inteligência. As limitações intelectuais constituem um dos indicadores a serem considerados em relação as outras quatro dimensões. Desse modo, a dimensão intelectual ocupa posição de destaque, mas não é condição suficiente para o diagnóstico de deficiência mental.

A Dimensão II - Comportamento adaptativo - compreende as habilidades conceituais, sociais e práticas (já referendadas anteriormente) aprendidas e utilizadas pelos sujeitos diariamente, no funcionamento de suas vidas. As habilidades adaptativas são avaliadas objetivamente por meio de medidas padronizadas presentes nas escalas de comportamento adaptativo. Carneiro (2008) tece uma crítica afirmando a fragilidade do caráter inovador do discurso do Sistema 2002, que não se sustenta se não houver dados mensuráveis e quantificáveis para a avaliação e, assim, propõe uma avaliação quantitativa de uma dimensão constituída por elementos subjetivos, interativos e contextuais.

A Dimensão III - Participação, interações e papéis sociais - refere-se à importância da participação do sujeito na comunidade e às interações estabelecidas com os mundos material e social. A observação direta das atividades cotidianas reflete melhor a participação e a interação do sujeito; quanto maior for essa participação e essa interação, maiores as possibilidades de exercer papéis sociais valorizados.

A Dimensão IV - Saúde - defende as condições de saúde física e mental e os fatores etiológicos, influenciando o funcionamento humano e os seus efeitos, podendo ser, ao mesmo tempo, amplamente facilitadores e inibidores da participação dos sujeitos em sociedade. Dessa forma, afirma a necessidade de serem considerados tais aspectos no diagnóstico de deficiência mental.

A Dimensão V - Contextos - refere-se às condições de vida cotidiana. Essa dimensão é avaliada principalmente por meio das oportunidades propiciadas aos su- 
jeitos em relação à vida, à educação, ao trabalho, à recreação e ao lazer. Além disso, objetiva identificar os fatores no ambiente capazes de estimular e melhorar o bem-estar, considerando, em especial, a saúde, a segurança pessoal e financeira, o conforto material, as atividades comunitárias e cívicas, o lazer e a recreação.

A definição de deficiência mental, proposta no Sistema 2002, a partir de uma concepção multidimensional - cinco dimensões -, é considerada um avanço em relação à definição dos outros manuais, por considerar a relação, a interação e a vivência dos sujeitos nessas diferentes dimensões. Outro ponto de divergência refere-se à classificação. No Sistema 2002, a classificação é baseada nas intensidades de apoio enquanto, no CID-10 e no DSM-IV, a classificação é realizada com base nos escores de quociente de inteligência (QI).

No CID-10, a classificação é realizada por meio de seis categorias, a saber: F70 Retardo Mental Leve (QI na faixa de 50 a 69); F7l Retardo Mental Moderado (QI usualmente na faixa de 35 a 49); F72 Retardo Mental Grave (QI em média na faixa de 20 a 34); F73 Retardo Mental Profundo (QI abaixo de 20); F78 Outro Retardo Mental (nesta classificação a avaliação, por meio de procedimentos usuais, está prejudicada, como, por exemplo, diante da necessidade de se avaliar um indivíduo cego ou surdo; dessa forma, ele é classificado em Outro Retardo Mental) e F79 Retardo Mental Não Especificado (nesta categoria há evidência de retardo mental, mas as informações disponíveis são insuficientes para designar uma das categorias).

No DSM-IV, a classificação é realizada por meio de cinco categorias. Neste manual, encontramos explicitamente uma previsibilidade sobre as possibilidades de existência dos sujeitos. A respeito das habilidades acadêmicas, para os sujeitos diagnosticados com Retardo Mental Leve (QI de 50-55 a aproximadamente 70), o DSM-IV prevê que, "ao final da adolescência, podem atingir habilidades acadêmicas equivalentes aproximadamente às da sexta série" (DSM-IV, 2002, p. 74). Tal previsibilidade varia conforme o grau de deficiência, ou seja, se o sujeito apresenta um quadro de Retardo Mental Moderado (QI de 35-40 a 50-55), "suas habilidades acadêmicas provavelmente não progredirão além do nível da segunda série" (DSM-IV, 2002, p.75). Os sujeitos pertencentes a um quadro de Retardo Mental Grave (QI de 20-25 a 35-40) "beneficiam-se apenas em um grau limitado de matérias escolares, tais como familiaridade com o alfabeto e contagem simples" (DSM-IV, 2002, p. 75). Para o grupo sob a classificação de Retardo Mental Profundo (QI abaixo de 20 ou 25) e Retardo Mental, Gravidade Inespecificada (quando existe forte suspeita, mas a inteligência não é possível de ser testada por instrumentos padronizados), as previsibilidades acadêmicas não são mencionadas. Poder-se-ia supor essa inexistência apoiada na ideia de que o processo de escolarização não seja possível para esses sujeitos. É possível observar a forte compatibilidade entre os dois manuais; estes são apresentados de forma muito semelhante tanto na nomenclatura utilizada para cada categoria, quanto nos índices de quociente de inteligência estabelecidos em cada uma delas.

O programa diagnóstico dos manuais constitui uma operação de classificação e distribuição dos sintomas em quadros regulares de transtornos e é a aplicação do conjunto sobre o relato 
pontual do indivíduo que determina sua nomeação e seu tratamento. Não é incomum observar, nas unidades de saúde ou mesmo nas escolas, que o nome do paciente ou do aluno seja substituído por sua classificação diagnóstica - estranha nomeação dos indivíduos que põe em relevo o lugar que ocupam na escala normal. (GUARIDO, 2007, p. 158)

Quanto ao Sistema 2002, a classificação é realizada por meio do sistema ILEP, isso significa que existem quatro categorias de classificação: intermitentes, limitadas, extensivas ou penetrantes (ILEP) e estas independem do QI apresentado pelo sujeito. Neste caso, a classificação é feita com base nas intensidades de apoios necessários ao indivíduo. São considerados diferentes fatores tais como: "(a) duração no tempo (quanto tempo um apoio é necessário); (b) frequência no tempo (com que frequência um apoio é necessário); (c) locais em que o apoio é necessário; (d) recursos necessários para os apoios; (por exemplo, custo, pessoal, perícia), e (e) grau de invasão na vida da pessoa." (AAMR, 2006, p. 103)

\section{Possíveis leituras sobre os manuais diagnósticos e seus efeitos na escolarização}

Apesar dos avanços observados no discurso do Sistema 2002, em especial, a respeito da concepção multidimensional e a classificação relacionada à intensidade dos sistemas de apoio, uma leitura mais ampla e contextual ainda é pouco explorada quando se refere ao diagnóstico. Este acaba por ser realizado por instrumentos mensuráveis e quantificáveis como os testes psicométricos de inteligência e escalas de comportamento adaptativo. Em vista disso, "na concepção de deficiência mental proposta pela AAMR, o foco ainda é o sujeito e suas limitações. Daí a necessidade de se quantificar os limites e definir a intensidade e as modalidades de apoio". (CARNEIRO, 2008, p. 24). Nesse sentido, apesar de alguns movimentos sinalizarem um esforço na construção de um conceito mais amplo e contextual da deficiência mental, a proposição do Sistema 2002 ainda apoia-se num discurso e numa lógica classificatória que tendencialmente dá prioridade ao plano individual e intrínseco do sujeito. O foco centra-se no sujeito e no seu comportamento, e não no conjunto de fatores e condições por meio dos quais o sujeito se produz.

A profusão das categorias diagnósticas, consideradas as ponderações precedentes, pode ser considerada um fruto da manifestação do pensamento científico moderno e dos pressupostos que o sustentam. Vasconcellos (2007), ao percorrer historicamente a organização do pensamento grego e medieval, demonstra a configuração do pensamento do homem moderno. Apresenta pontos importantes, fundadores da constituição do pensamento científico, e elementos característicos do que a autora define como "paradigma tradicional da ciência", refletido por meio de três pressupostos epistemológicos fundamentais:

1. O pressuposto da simplicidade: a crença em que, separando-se o mundo complexo em partes, encontram-se elementos simples, em que é preciso separar as partes para entender o todo, ou seja, o pressuposto de que "o microscópio é simples". Daí decorre, entre outras coisas, a atitude de análise e a busca de relações causais lineares. 
2. O pressuposto da estabilidade do mundo: a crença em que o mundo é estável, ou seja, em que "o mundo já é". Ligados a este pressuposto estão a crença na determinação - com consequente previsibilidade dos fenômenos - e a crença na reversibilidade - com consequente controlabilidade dos fenômenos.

3. O pressuposto da objetividade: a crença em que "é possível conhecer objetivamente o mundo tal como ele é na realidade" e a exigência da objetividade como critério de cientificidade. Daí decorrem os esforços para colocar entre parênteses a subjetividade do cientista, para atingir o universo, ou versão única do conhecimento. (VASCONCELLOS, 2007, p. 69)

Perceptivelmente, estes pressupostos da ciência tradicional compõem a lógica de sustentação dos sistemas classificatórios. A ação diagnóstica e classificatória é pautada na necessidade de se conhecer objetivamente o fenômeno em questão, de detalhá-lo minuciosamente, afim de que se possa construir uma previsibilidade "suas habilidades acadêmicas provavelmente não progredirão além do nível da segunda série" (DSM-IV, 2002, p. 75), ou seja, um prognóstico sobre as possibilidades de existência dos sujeitos. Dessa forma, intenciona-se controlar o fenômeno. Conhecer para prever e controlar. O Sistema 2002, por exemplo, referenda a importância de se conhecer o ambiente e identificar os fatores capazes de estimular os sujeitos com deficiência mental e garantir seu bem-estar. Destaca “... um dos aspectos mais importantes de qualquer ambiente é sua qualidade de estabilidade com aspectos associados de previsibilidade e controle." (AAMR, 2006, p. 56). Ou seja, se o ambiente é estável, conhecê-lo permite a previsão e o controle tanto do ambiente como do sujeito. É possível conhecer, para antecipar reações e comportamentos, controlá-los. Este paradigma considera, "por um lado a realidade de forma linear, fragmentada, como se fosse uma coleção de coisas e estável e, por outro, o sujeito que estuda essas questões é sempre externo a elas." (PELLANDA, 2009, p. 14)

A suposta previsibilidade permite a construção de explicações causais lineares, tendencialmente simples e reduzidas a atribuições de causa e efeito "... não aprende porque é deficiente mental...”. Estes pressupostos representam a construção do pensamento humano, configuram um paradigma, uma forma de compreensão do mundo e da experiência humana.

Como representação do pensamento humano, num determinado período histórico, essas características de objetividade, previsibilidade, controlabilidade, de relações causais lineares ganham visibilidade no campo escolar. Vivemos dentro da escola a necessidade diagnóstica e classificatória; buscam-se, nas diferentes categorizações, explicações sobre os modos de ser do sujeito já "preso" a sua classificação. Assim, "os critérios e procedimentos usados no processo de classificação determinam não somente os aspectos do indivíduo que serão focalizados (inteligência, conduta adaptativa, sociabilidade, etc.), mas principalmente as atitudes e práticas educativas com relação a ele." (NUNES; FERREIRA, 1993, p. 39)

A descrição comportamental de cada categoria produz uma imagem sobre quem são esses alunos; em geral, temos a reafirmação de uma representação homo- 
gênea, a qual impede percepções sobre as especificidades de cada um e a construção de experiências singulares. A descrição comportamental constrói e delimita práticas. Tais descrições pressupõem um aluno classificável, reduzido a um quadro de manifestações comportamentais; parecem não envolver os sentidos, os significados, as singularidades e a subjetividade. Conforme as contribuições de Pellanda (2009), tal prática encontra suas origens na ciência clássica e na atuação desta enquanto um conjunto de conhecimentos sem sujeito (ou um sujeito-fantasma), porque desconsidera a participação concreta deste na constituição da realidade, do aprender e de si mesmo.

Em vista disso, problematizo o uso dos diagnósticos na construção e delineamento das práticas pedagógicas. Reconheço sua presença e sua força no terreno escolar. O diagnóstico impulsionado pelas contribuições psicométricas, a partir de Binet, sempre encontrou, na escola, solo fértil para se desenvolver. Dessa forma, o uso das suas medições, em muito, determinaram a organização dos serviços em educação especial, principalmente, no que se refere ao encaminhamento de crianças com histórico de fracasso escolar para os espaços especializados - classes e escolas especiais.

Hoje, essa dimensão se reapresenta a partir da discussão sobre a necessidade, ou não, do diagnóstico clínico da deficiência mental para a frequência do aluno no Atendimento Educacional Especializado. No ano de 20ll, tivemos o abandono desta exigência. Os alunos com deficiência mental sem diagnóstico clínico puderam ser incluídos no Censo Escolar mediante avaliação e parecer do professor do Atendimento Educacional Especializado. Entendo essa medida como uma possibilidade oferecida à educação e à educação especial de apropriação ou (re)apropriação de seus domínios, apesar de reconhecer que a identificação desse aluno por parte dos educadores especializados não elimina os impasses decorrentes da complexidade da tarefa e da necessidade de investimento em formação de profissionais capazes de realizá-la.

\section{Referências}

ALMEIDA, M. A. Apresentação e análise das definições de deficiência mental propostas pela AAMR Associação Americana de Retardo Mental de 1908 e 2002. Revista de Educação, Campinas, n. 6, p. 33-48, 2004.

AMERICAN ASSOCIATION ON MENTAL RETARDATION - AAMR. Retardo mental: definição, classificação e sistemas de apoio. 10 ed. Porto Alegre: Artmed, 2006.

BRIDI, F.R. Processos de identificação e diagnóstico: os alunos com deficiência mental no contexto do atendimento educacional especializado. 2011. 210 f. + Anexos. Tese (Doutorado) - Universidade Federal do Rio Grande do Sul, Porto Alegre, 2011.

CARNEIRO, M. S. C. A deficiência mental como produção social: de Itard à abordagem histórico-cultural. In: BAPTISTA. C. (Org.). Inclusão e escolarização: múltiplas perspectivas. Porto Alegre: Mediação, 2006. p. $137-148$.

CARNEIRO, M. S. C. Deficiência mental como produção social: uma discussão a partir de histórias de vida de adultos com Síndrome de Down. Tese (Doutorado) - Programa de Pós Graduação em Educação, Faculdade de Educação, Universidade Federal do Rio Grande do Sul, UFRGS, Porto Alegre, 2007.

Adultos com Síndrome de Down: a deficiência mental como produção social. Campinas, São Paulo: Papirus, 2008.

CARVALHO, E. ; MACIEL, D. Nova concepção de deficiência mental segundo a American Association on Mental Retardation - AAMR: sistema 2002. In: Revista Temas em Psicologia da Sociedade Brasileira de Psicologia. v. 1l. n. 2. Sociedade Brasileira de Psicologia - SBP, 2003. p. 147-156. Disponível em: 〈http:// www.sbponline.org.br/revista2/vollln2/art07_t.htm>. Acesso em: 21 out. 2009. 
DINIZ, D.; MEDEIROS, M.; SQUINCA, F. Reflexões sobre a versão em português da classificação internacional de funcionalidade, incapacidade e saúde. In: Caderno de Saúde Pública. vol. 23, n. 10, Rio de Janeiro, 2007. p. 2507-2510.

DSM-IV-TR. Manual diagnóstico e estatístico de transtornos mentais. 4ed. Revista Porto Alegre: Artmed, 2002.

FARIAS, N. ; BUCHALLA, C. M. A Classificação internacional de funcionalidade, incapacidade e saúde: conceitos, usos e perspectivas. In: Revista Brasileira de Epidemiologia. São Paulo, vol. 8, n. 2, p. 187-193, 2005.

GARCIA, R. M. C. Política nacional de educação especial nos anos 2000: a formação de professores e a hegemonia do modelo especializado. In: Anais. V Seminário Nacional de Pesquisa em Educação Especial: formação de professores em foco. CD-ROM. São Paulo: UFES, UFRGS, UFSCar, 2009a.

GUARIDO, R. A medicalização do sofrimento psíquico: considerações sobre o discurso psiquiátrico e seus efeitos na educação. In: Educação e pesquisa. São Paulo, vol. 33, n. 1, p. 151-161, 2007.

MACHADO, A. M. Avaliação e fracasso: a produção coletiva da queixa escolar. In: AQUINO, J. G. Erro e fracasso na escola: alternativas teóricas e práticas. São Paulo: Summus, 1997. p. 73-90.

MICHELS, M. H. O instrumental, o gerencial e a formação à distância: estratégias para a reconversão docente na perspectiva da educação inclusiva. In: Anais. V Seminário Nacional de Pesquisa em Educação Especial: formação de professores em foco. CD-ROM. São Paulo: UFES, UFRGS, UFSCar, 2009a.

MICHELS, M. H. Paradoxo da formação docente na política da educação inclusiva do Estado de Santa Catarina: a perspectiva clínica como sustentação do trabalho escolar. In: BAPTISTA, C. ; JESUS, D. M. Avanços em Políticas de Inclusão: o contexto da educação especial no Brasil e em outros países. Porto Alegre: Mediação, 2009b., p. 139-152.

MOYSÉS, M. A. A. A institucionalização invisível: crianças que não-aprendem-na-escola. Edição revista e ampliada. Campinas: Mercado de Letras, 2008.

NUNES, L. ; FERREIRA, J. Deficiência mental: o que as pesquisas têm revelado. In: Revista em aberto. Ano 13. n. 60. Brasilia, DF: MEC/INEP, 1993.

ORGANIZAÇÃO MUNDIAL DA SAÚDE. Classificação de transtornos mentais e de comportamento da CID-10 - Descrições clínicas e diretrizes diagnósticas. Porto Alegre: Artes Médicas, 1993.

ORGANIZAÇÃO MUNDIAL DA SAÚDE. Classificação Internacional de Funcionalidade, Incapacidade e Saúde - CIF. Classificação detalhada com definições. Todas as categorias com suas definições, inclusões e exclusões. OMS, 2003.

PATTO, M. H. S. A produção do fracasso escolar: histórias de submissão e rebeldia. $3^{a}$ ed. São Paulo: Casa do Psicólogo, 2010.

PELLANDA, N. M. C. Maturana e a educação. Coleção pensadores \& educação. Belo Horizonte: Autêntica, 2009

VASQUES, C. Construções em torno de um vazio: uma leitura sobre o diagnóstico e seus "modos de usar" na escolarização de sujeitos com autismo e psicose infantil. In: BAPTISTA, C. R.; JESUS, D. M. (Orgs.). Conhecimento e margens: ação pedagógica e pesquisa em educação especial. Porto Alegre: Mediação, 2009. p. 11-26

. Alice na biblioteca mágica: uma leitura sobre o diagnóstico e a escolarização de crianças com autismo e psicose infantil. 2008. 195 f. + Anexos. Tese (Doutorado) - Programa de Pós-Graduação em Educação, Faculdade de Educação, Universidade Federal do Rio Grande do Sul, Porto Alegre, 2008.

VASCONCELLOS, M. J. E. Pensamento sistêmico: o novo paradigma da ciência. $6^{a}$ ed. Campinas: Papirus, 2007.

\section{Notas}

${ }^{1}$ Cabe destacar que a décima edição deste manual diagnóstico da deficiência mental passou por recente revisão conceitual que foi apresentada a partir de nova publicação nos Estados Unidos, ainda sem tradução para o português. "Intellectual Disability: Definition, classification, and Systems of Supports" (1lth edition, 2010). Neste manual, segundo Veltrone (2011), a definição de retardo mental permanece a mesma apresentada em 2002 apesar da troca de terminologia para Intellectual Disabilities (deficiência intelectual). Segundo as orientações da própria 
Associação, o termo refere o mesmo grupo de pessoas que eram elegíveis para o diagnóstico de retardo mental, devendo ser tratado como sinônimos. E, ainda, merece destaque a alteração do nome da Associação Americana de Retardo Mental (AAMR) que passou a se chamar American Association on Intellectual Developmental Disabilities (AAIDD).

${ }^{2}$ As Escalas de Inteligência disponíveis e utilizadas são: WISC - III; WAIS - III; Stanford-Binet - IV; Sistema de Avaliação Cognitiva e Bateria Kaufman para avaliação de crianças. Destaco que as escalas Weshsler (WISC e WAIS) e Satanford-Binet são os instrumentos mais frequentemente utilizados para avaliar a inteligência (AAMR, 2002)

${ }^{3}$ Era inglês e primo de Charles Darwin.

${ }^{4} \mathrm{O}$ comportamento adaptativo é verificado por meio da Escala de Comportamento Adaptativo de Vineland; Escala de Comportamento Adaptativo (ABS - Adaptive Behavior Scales) da AAMR; Escala de Comportamento Independente (Scales of Independent Behavior); do Teste Abrangente do Comportamento Adaptativo - Revisado (Comprehensive Testo of Adaptative Behavior - Revised) e do Sistema de Avaliação do Comportamento Adaptativo (Adaptive Behavior Assessmente System), (AAMR, 2006).

\section{Correspondência}

Fabiane Romano de Souza Bridi - Universidade Federal de Santa Maria, Centro de Educação. Avenida Roraima, prédio 16, Camobi, CEP: 97105-900 - Santa Maria, Rio Grande do Sul - Brasil.

E-mail:fabianebridi@gmail.com - baptistacaronti@yahoo.com.br

Recebido em 12 de agosto de 2013

Aprovado em 03 de abril de 2014 